On Local Fusion Graphs of Finite Coxeter Groups

Ballantyne, John J

2013

MIMS EPrint: 2011.2

Manchester Institute for Mathematical Sciences

School of Mathematics

The University of Manchester

\footnotetext{
Reports available from: http://eprints.maths.manchester.ac.uk/

And by contacting: The MIMS Secretary

School of Mathematics

The University of Manchester

Manchester, M13 9PL, UK
} 


\title{
On Local Fusion Graphs of Finite Coxeter Groups
}

\author{
John J. Ballantyne
}

August 29, 2013

The University of Manchester, School of Mathematics, Alan Turing Building, Oxford Road, Manchester, M13 9PL

john.ballantyne@manchester.ac.uk

Mathematical Subject Classification 20F55

Key Words: Group, Coxeter, Graph, Involution

\begin{abstract}
Given a finite group $G$ and $G$-conjugacy class of involutions $X$, the local fusion graph $\mathcal{F}(G, X)$ has $X$ as its vertex set, with $x, y \in X$ joined by an edge if, and only if, $x \neq y$ and the product $x y$ has odd order. In this note we investigate such graphs when $G$ is a finite Coxeter group, addressing questions of connectedness and diameter. In particular, our results show that local fusion graphs may have an arbitrary number of connected components, each with arbitrarily large diameter.
\end{abstract}




\section{Introduction}

Let $G$ be a finite group, with $X$ a $G$-conjugacy class of involutions. We define the local fusion graph $\mathcal{F}(G, X)$ by taking $X$ to be our vertex set, and joining vertices $x, y \in X$ with an edge if, and only if, $x \neq y$ and the product $x y$ has odd order. These graphs are so called because given two elements $x, y \in X$, and a path

$$
x=x_{0} \rightarrow x_{1} \rightarrow \cdots \rightarrow x_{m}=y
$$

from $x$ to $y$ in $\mathcal{F}(G, X)$, there exists an element $g_{1} g_{2} \cdots g_{m-1}$ which conjugates $x$ to $y$, where $g_{i} \in\left\langle x_{i-1}, x_{i}\right\rangle$ for $1 \leq i \leq m$.

Local fusion graphs arise in a number of situations. For example, they can be used to construct Fischer's sporadic simple groups $F i_{22}, F i_{23}$ and $F i_{24}^{\prime}$ [12]. Also, both Glauberman's $Z^{*}$-theorem (for involutions - see Theorem 1 of [13]) and the Baer-Suzuki theorem (for the prime 2 - see Theorem 8.2 of [14]) may be restated in terms of local fusion graphs, with the connection to the latter result being further explored in [2], where it is shown that the local fusion graphs of finite simple groups are connected. While in [3] the usefulness of local fusion graphs in a computational context is highlighted, through a method to compute involution centralisers.

In this note we investigate the local fusion graphs of finite Coxeter groups, addressing two fundamental questions: when are such graphs connected, and given a connected graph, what can we say about its diameter? This work may be viewed as a sequel to [1], where these issues were resolved for the local fusion graphs of finite symmetric groups. Similar problems have been tackled for other graphs which are closely related to local fusion graphs. For example, commuting involution graphs have a conjugacy class of involutions as their vertex set, with distinct vertices joined by an edge if, and only if, the relevant involutions commute. In [4], [5] and [6], properties of these graphs are investigated for a variety of groups, including finite Coxeter groups. Even more closely related to the graphs of the present article are $S_{3}$-involution graphs, where vertices are again involutions, and adjacent vertices must have product order 3 . These objects were studied in [10] for some finite projective special linear groups of dimension 2 , and utilised further in [11].

Recall that the finite irreducible Coxeter groups have been classified (see for example [15]), and consist of the three classical families $C\left(A_{n}\right), C\left(B_{n}\right)$ and $C\left(D_{n}\right)$, along with the exceptional groups $C\left(E_{6}\right), C\left(E_{7}\right), C\left(E_{8}\right), C\left(F_{4}\right), C\left(H_{3}\right)$, $C\left(H_{4}\right)$ and $C\left(I_{n}\right)$. The structure of these groups is shown below. 


$$
\begin{aligned}
& C\left(A_{n}\right) \cong \operatorname{Sym}(n+1), \quad(n \geq 1) ; \\
& C\left(B_{n}\right) \cong 2^{n}: \operatorname{Sym}(n), \quad(n \geq 2) ; \\
& C\left(D_{n}\right) \cong 2^{n-1}: \operatorname{Sym}(n), \quad(n \geq 4) ; \\
& C\left(E_{6}\right) \cong \mathrm{GO}_{6}^{-}(2) \text {; } \\
& C\left(E_{7}\right) \cong 2 . \operatorname{Sp}_{6}(2) \text {; } \\
& C\left(E_{8}\right) \cong 2 . \mathrm{O}_{8}^{+}(2) .2 \text {; } \\
& C\left(F_{4}\right) \cong 2_{+}^{1+4} \cdot 3^{2} \cdot 2^{2} \\
& C\left(H_{3}\right) \cong 2 \times \operatorname{Alt}(5) \text {; } \\
& C\left(H_{4}\right) \cong\left(\mathrm{SL}_{2}(5) \circ \mathrm{SL}_{2}(5)\right): 2 \text {; } \\
& C\left(I_{n}\right) \cong \operatorname{Dih}(2 n) \text {. }
\end{aligned}
$$

Here, $\operatorname{Alt}(n)$ and $\operatorname{Sym}(n)$ denote the alternating and symmetric groups on $n$ letters respectively, and we have used AtLAs notation [9]. Let us now summarise our main results. Firstly, we have the classical families $C\left(B_{n}\right)$ and $C\left(D_{n}\right)$. The notation used here will be explained in Section 2.

Theorem 1.1. Suppose $G=C\left(B_{n}\right), n \geq 2$ and $X$ is a $G$-conjugacy class of involutions. Write $G=N: H$ where $N \cong 2^{n}$ and $H \cong \operatorname{Sym}(n)$. If $X \subset N$, then $\mathcal{F}(G, X)$ is totally disconnected, while if $X \not \subset N$ then $\mathcal{F}(G, X)$ is connected, unless $n=2 m$ and $X=t^{G}$, where

$$
t=(\stackrel{+}{1}, \stackrel{+}{2})(\stackrel{+}{3}, \stackrel{+}{4}) \cdots\left(2 m^{+}-1,2 \stackrel{+}{m}\right) .
$$

In the latter case $\mathcal{F}(G, X)$ has exactly two connected components, unless $n=4$, in which case $\mathcal{F}(G, X)$ is totally disconnected with 12 vertices.

Theorem 1.2. Suppose $G=C\left(D_{n}\right), n \geq 4$ and $X$ is a $G$-conjugacy class of involutions. Write $G=N: H$ where $N \cong 2^{n-1}$ and $H \cong \operatorname{Sym}(n)$. If $X \subset N$, then $\mathcal{F}(G, X)$ is totally disconnected, while if $X \not \subset N$ then $\mathcal{F}(G, X)$ is connected.

If we have a connected local fusion graph $\mathcal{F}(G, X)$, we denote its diameter by $\operatorname{Diam} \mathcal{F}(G, X)$.

Theorem 1.3. Suppose $G=C\left(B_{n}\right)$, where $n \geq 4$.

(i) If $n$ is even, then there exists a $G$-conjugacy class of involutions $X$ such that $\operatorname{Diam} \mathcal{F}(G, X)=n-1$. Moreover, if $X^{\prime}$ is any other $G$-conjugacy class of involutions such that $\mathcal{F}\left(G, X^{\prime}\right)$ is connected, then

$$
\operatorname{Diam} \mathcal{F}\left(G, X^{\prime}\right) \leq n-1
$$

(ii) If $n$ is odd, then there exists a $G$-conjugacy class of involutions $X$ such that $\operatorname{Diam} \mathcal{F}(G, X)=n-2$. Moreover, if $X^{\prime}$ is any other $G$-conjugacy class of involutions such that $\mathcal{F}\left(G, X^{\prime}\right)$ is connected, then

$$
\operatorname{Diam} \mathcal{F}\left(G, X^{\prime}\right) \leq n-2 \text {. }
$$


We shall see from Proposition 2.1 that, with possibly just one exception, the local fusion graphs of $C\left(D_{n}\right)$ are isomorphic to local fusion graphs of $C\left(B_{n}\right)$. Thus Theorem 1.3 also provides bounds on the diameters of the local fusion graphs of $C\left(D_{n}\right)$. As a consequence of our work on $C\left(B_{n}\right)$ we have the following more general result concerning local fusion graphs of finite groups.

Theorem 1.4. For any given $r, m \in \mathbb{N}$, there exists a finite group $G$ with conjugacy class of involutions $X$ such that $\mathcal{F}(G, X)$ has exactly $m$ connected components, each of which has diameter $r$.

Theorems 1.3 and 1.4 contrast with many results concerning the diameter of graphs related to local fusion graphs. For example, in [6] it is shown that for finite symmetric groups the diameter of commuting involution graphs is at most 4, while in [4] it is proved that for any other finite irreducible Coxeter group the diameter of a commuting involution graph is at most 5. Also, in [5], we find analysis of the commuting involutions graphs of the majority of the sporadic simple groups, and it is shown that their diameters are at most 4 . For the $S_{3}$-involution graphs studied in [10], it is shown that their diameter is 3 . Additionally, for local fusion graphs themselves, in [1] we find that for finite symmetric groups (excluding some small exceptions) local fusion graphs have diameter 2. It is therefore worthwhile to note that Theorem 1.3 demonstrates that no such absolute bounds exist for the diameters of local fusion graphs of finite Coxeter groups of type $B_{n}$ (and $D_{n}$ ).

Our final result concerns the exceptional Coxeter groups. If $X$ is a $G$ conjugacy class, then for $x \in X$ and $i \in \mathbb{N}$ we define

$$
\Delta_{i}(x)=\{y \in X \mid d(x, y)=i\}
$$

where $d$ is the usual graph-theoretic distance metric, and refer to the $i$-th disc of $\mathcal{F}(G, X)$ (with respect to $x$ ).

Theorem 1.5. Suppose that $G$ is an exceptional finite Coxeter group and $X$ is a G-conjugacy class of involutions.

(i) If $G=C\left(I_{n}\right)$, then $\mathcal{F}(G, X)$ is either a disjoint union of complete graphs, or consists of a single vertex (see Lemma 3.1).

(ii) If $G=C\left(E_{6}\right)$, then $\operatorname{Diam} \mathcal{F}(G, X)=2$.

(iii) If $G=C\left(E_{7}\right)$ and $|X|>1$, then $\operatorname{Diam} \mathcal{F}(G, X)=2$.

(iv) If $G=C\left(E_{8}\right)$ and $|X|>1$, then $\operatorname{Diam} \mathcal{F}(G, X) \leq 3$.

(v) If $G=C\left(F_{4}\right)$ and $|X|>1$, then either $\mathcal{F}(G, X)$ is totally disconnected with 18 vertices, or $\operatorname{Diam} \mathcal{F}(G, X) \leq 3$.

(vi) If $G=C\left(H_{3}\right)$ and $|X|>1$, then $\operatorname{Diam} \mathcal{F}(G, X)=2$.

(vii) If $G=C\left(H_{4}\right)$ and $|X|>1$, then $\operatorname{Diam} \mathcal{F}(G, X) \leq 3$. 
For cases (ii)-(vii), the sizes of the discs $\Delta_{i}(t)$ are given in Table 1 .

Finally, the author would like to thank the referees who have reviewed various incarnations of this paper. Their suggestions greatly improved the final outcome.

\section{The Classical Groups}

We now begin our analysis of the local fusion graphs of the finite classical Coxeter groups. Since the groups $C\left(A_{n}\right)$ have been tackled previously in [1], here we concentrate on the groups $C\left(B_{n}\right)$ and $C\left(D_{n}\right)$. The Coxeter group $C\left(B_{n}\right)$ may be considered as the group of signed permutations of $n$ objects (see [4] or [15]). Let $\operatorname{Sym}(n)$ act on the set $\Omega=\{1, \ldots, n\}$, and define the $i$-th 'sign change' to be the element which sends $i$ to $-i$ and fixes all other $j \in \Omega$. The set of all such elements generates an elementary abelian group of order $2^{n}$, and $C\left(B_{n}\right)$ is isomorphic to the semidirect product of this group with $\operatorname{Sym}(n)$. If we wish to emphasise the set upon which $C\left(B_{n}\right)$ acts, we may write $C\left(B_{\Omega}\right)$. For $n \geq 4, C\left(D_{n}\right)$ is the subgroup of index 2 of $C\left(B_{n}\right)$ generated by $\operatorname{Sym}(n)$ and the elements of the elementary abelian subgroup involving an even number of sign changes. A convenient way of expressing the elements of $C\left(B_{n}\right)$ is to write a permutation in $\operatorname{Sym}(n)$, including 1-cycles, along with a plus or minus sign above each $i$, and say $i$ is positive or negative respectively, for example

$$
z=(\stackrel{+}{1}, \overline{3}, \stackrel{+}{2})(\overline{4}) \in C\left(B_{4}\right) .
$$

By convention we read the sign first, so $z(1)=3, z(2)=1, z(3)=-2$ and $z(4)=-4$. Given a cycle $\rho \in C\left(B_{n}\right)$, we say $\rho$ is positive or negative depending on whether the number of minus signs above its elements is even or odd, respectively. The signed cycle type of an element of $C\left(B_{n}\right)$ is defined to be the cycle type of the element, including 1-cycles, with a plus or minus over each cycle, depending on whether the cycle is positive or negative, respectively.

We may now state the following result, found in [8], which characterises the conjugacy classes of $C\left(B_{n}\right)$ and $C\left(D_{n}\right)$.

Proposition 2.1. $\quad$ (i) Elements of $C\left(B_{n}\right)$ are conjugate if and only if they have the same signed cycle type.

(ii) Conjugacy classes in $C\left(D_{n}\right)$ are parameterised by signed cycle type, with one class for each signed cycle type except in the case where the signed cycle type contains only even length, positive cycles. In the latter case there are two classes for each signed cycle type, distinguished by the number of minus signs modulo 4.

As we are considering $C\left(D_{n}\right)$ as a subgroup of $C\left(B_{n}\right)$, Proposition 2.1 (ii) tells us that for $x \in C\left(D_{n}\right)$ we have $x^{C\left(D_{n}\right)}=x^{C\left(B_{n}\right)}$, unless $x$ has only even length, positive cycles. Let us now establish some notation. Recall that for an element $\sigma \in \operatorname{Sym}(n)$, the support of $\sigma$ is defined to be $\operatorname{supp}(\sigma)=\Omega \backslash \operatorname{fix}(\sigma)$. We 
now extend this notion to $C\left(B_{n}\right)$. Given $x \in C\left(B_{n}\right)$, we define the $S$-support of $x, \operatorname{supp}_{S}(x)$, to be the support of the corresponding element of $\operatorname{Sym}(n)$. In addition, we define the $C$-support of $x, \operatorname{supp}_{C}(x)$, to be $\Omega \backslash$ fix $(x)$. Here $S$ and $C$ stand for 'symmetric' and 'Coxeter' respectively. To illustrate, if we again take

$$
z=(\stackrel{+}{1}, \overline{3}, \stackrel{+}{2})(\overline{4}),
$$

then $\operatorname{supp}_{S}(z)=\{1,2,3\}$, while $\operatorname{supp}_{C}(z)=\{1,2,3,4\}$ (since $z(4)=-4$ ). For brevity, given two elements $x$ and $y$ of $C\left(B_{n}\right)$ we shall write

$$
\Delta_{x, y}=\operatorname{supp}_{S}(x) \cup \operatorname{supp}_{S}(y) .
$$

We define the weight of $x$, denoted $w(x)$, to be the number of negative signs in $x$. If $X$ is a conjugacy class of involutions of $C\left(B_{n}\right)$ or $C\left(D_{n}\right)$, then given two elements $x, y \in X$ we define the 1-weight of the product $x y$, denoted $w_{1}(x y)$, to be the number of negative 1-cycles $(\bar{i})$ in $x y$ such that $i \notin \Delta_{x, y}$. For example, suppose that

$$
x=(\stackrel{+}{1}, \stackrel{+}{2})(\overline{3})(\overline{4})(\stackrel{+}{5})(\stackrel{+}{6})
$$

and

$$
y=(\overline{1}, \overline{2})(\overline{3})(\stackrel{+}{4})(\overline{5})(\stackrel{+}{6}) .
$$

Then $w(x)=2$ and $w(y)=4$. Also,

$$
x y=(\overline{1})(\overline{2})(\stackrel{+}{3})(\overline{4})(\overline{5})(\stackrel{+}{6})
$$

which has 4 negative 1-cycles, so $w(x y)=4$, but since $1,2 \in \Delta_{x, y}$ we have $w_{1}(x y)=2$.

We shall require the following easy lemma, which holds in general for local fusion graphs.

Lemma 2.2. Let $G$ be a group with $G$-conjugacy class of involutions $X$. Suppose $x, y \in X$ are such that $d(x, y)=k$ in $\mathcal{F}(G, X)$, where $d$ is the usual graph metric. Then for any $g \in C_{G}(x), d\left(x, y^{g}\right)=k$.

Proof. This follows immediately from the observation that elements of $C_{G}(x)$ induce graph automorphisms of $\mathcal{F}(G, X)$ which leave $x$ fixed.

We now set $G=C\left(B_{n}\right)$ where $n \geq 3$, and work towards proving Theorems 1.1 and 1.3. Clearly a $G$-conjugacy of involutions which lies in the elementary abelian normal 2-subgroup of $G$ will yield a totally disconnected local fusion graph. A more interesting collection of local fusion graphs of $G$ are those which have as vertex set a $G$-conjugacy class of signed transpositions, that is, elements of $G$ which contain exactly one 2 -cycle. Let $X$ be such a $G$-conjugacy class. Our next lemma tells us precisely when two elements of $X$ are adjacent in $\mathcal{F}(G, X)$.

Lemma 2.3. If $x, y \in X$, then $x$ and $y$ are adjacent in $\mathcal{F}(G, X)$ if, and only if, $\left|\Delta_{x, y}\right|=3$ and $w_{1}(x y)=0$. 
Proof. Suppose that $x$ and $y$ are adjacent in $\mathcal{F}(G, X)$. Then since $x$ and $y$ contain only one 2-cycle each, the product $x y$ must have order 3 , and we must certainly have $\left|\Delta_{x, y}\right|=3$. Furthermore, if $w_{1}(x y) \geq 1$, then $x y$ must contain negative 1-cycles, and consequently the product order must be divisible by 2 . This contradicts $x y$ having order 3 , and so $w_{1}(x y)=0$.

Now suppose that $\left|\Delta_{x, y}\right|=3$ and $w_{1}(x y)=0$. By the latter assumption, any 1-cycle $(i)$ in the product $x y$, where $i \in \Omega \backslash \Delta_{x, y}$, must be positive, and hence have order 1 . So we need only concern ourselves with the 3 -cycle of $x y$. We may without loss of generality assume that, without signs, we have $x=(1,2)(3)$ and $y=(1,3)(2)$. Since $x$ and $y$ are $G$-conjugate (and the 1-cycles of $x$ and $y$ outside $\Delta_{x, y}$ have the same signs), we can apply Proposition 2.1 to deduce that the 1-cycles (3) and (2) must have the same sign.

If the signs of $x$ are the same as those of $y$, we get

$$
x y=(\stackrel{+}{1}, \stackrel{+}{2}, \stackrel{+}{3})
$$

which clearly has order 3 , so $x$ and $y$ are adjacent in $\mathcal{F}(G, X)$. On the other hand, any other valid signing of $x$ and $y$ will a yield a 3 -cycle with two negative signs and one positive, such as $x y=(\overline{1}, \overline{2},++\underset{3}{+}$. An easy check shows that such elements also have order 3 . Thus $x$ and $y$ are adjacent in any case.

An easy consequence of Lemma 2.3 is that the local fusion graphs of $C\left(B_{n}\right)$ corresponding to $G$-conjugacy classes of signed transpositions are also $S_{3}$-involution graphs, as defined in [10]. We now prove a lemma which gives us a lower bound on the distance between two $G$-conjugate signed transpositions in $\mathcal{F}(G, X)$.

Lemma 2.4. Suppose that $x, y \in X$, with $x \neq y$. If $w_{1}(x y)=k$, then $d(x, y) \geq$ $k+1$.

Proof. Firstly, note that if $x$ is not connected to $y$ in $\mathcal{F}(G, X)$, then by convention $d(x, y)=\infty$, and the result holds. So suppose $x$ is connected to $y$, and consider a shortest path from $x$ to $y$ in $\mathcal{F}(G, X)$, say

$$
x=x_{0} \rightarrow x_{1} \rightarrow x_{2} \rightarrow \cdots \rightarrow x_{r}=y .
$$

By Lemma 2.3, for $1 \leq i \leq r$ we have $w_{1}\left(x_{i-1} x_{i}\right)=0$ and $\left|\Delta_{x_{i-1}, x_{i}}\right|=3$. Therefore, for each $i$, the signed 1-cycles of $x_{i}$ with $C$-support outside $\Delta_{x_{i-1}, x_{i}}$ are identical to those of $x_{i-1}$, and $x_{i}$ contains exactly one signed 1-cycle which does not appear in $x_{i-1}$. Consequently $w_{1}\left(x x_{i}\right) \leq w_{1}\left(x x_{i-1}\right)+1$, for $1 \leq i \leq r$. Since $w_{1}\left(x x_{1}\right)=0$, for all $i \leq k$ we must have $w_{1}\left(x x_{i}\right)<k$. As $w_{1}(x y)=\bar{k}$, it follows that $r \geq k+1$.

Given $x, y \in X$, then since $\left|\operatorname{supp}_{S}(x)\right|=\left|\operatorname{supp}_{S}(y)\right|=2$, it must be the case that $\left|\Delta_{x, y}\right|=2,3$ or 4 . Lemmas 2.5 and 2.7 address each of these possibilities, and provide us with straightforward expressions for the distance between $x$ and $y$ in $\mathcal{F}(G, X)$ in terms of the 1 -weight of the product $x y$. 
Lemma 2.5. Suppose $x, y \in X$, where $x \neq y$ and $\left|\Delta_{x, y}\right|=2$ or 3 . Then $d(x, y)=w_{1}(x y)+1$, unless $\left|\Delta_{x, y}\right|=2$ and $w_{1}(x y)=0$, in which case $d(x, y)=$ 2 .

Proof. First suppose that $\left|\Delta_{x, y}\right|=2$, and that $w_{1}(x y)=k$. Note that now Proposition 2.1 implies that $k$ must be even, so write $k=2 m$. If $m=0$, then since $x \neq y$ without loss of generality the 2-cycles of $x$ and $y$ must be $(\stackrel{+}{1}, \stackrel{+}{2})$ and $(\overline{1}, \overline{2})$, and using Lemma 2.3 we may easily find an element which is adjacent to both $x$ and $y$ in $\mathcal{F}(G, X)$. So assume that $m \geq 1$, and consequently we must have $n \geq 4$. If $x$ and $y$ agree on any signed 1-cycles, then in the product $x y$ these will be positive 1-cycles, which have order 1. It therefore suffices to ignore these 1cycles and consider $x$ and $y$ as elements of $C\left(B_{\Sigma}\right)$, where $\Sigma=\Delta_{x, y} \cup \operatorname{supp}_{C}(x y)$, and prove the result in this context. So, without loss of generality we assume that $n=\left|\Delta_{x, y}\right|+w_{1}(x y)$. Using the vertex-transitivity of $G$ on $\mathcal{F}(G, X)$, and Proposition 2.1, we may assume that $x$ and $y$ are labelled so that

$$
x=(\stackrel{+}{1}, \stackrel{+}{2})(\overline{3})(\overline{4}) \cdots(m+2)(m+3) \cdots\left(2 m^{+}+2\right)
$$

and

$$
y=(\stackrel{\epsilon}{1}, \stackrel{\epsilon}{2})(\stackrel{+}{3})(\stackrel{+}{4}) \cdots(m+2)\left(m_{+}^{+} 3\right) \cdots\left(2 m^{-}+2\right),
$$

where $\epsilon \in\{+,-\}$. By Lemma 2.4, $d(x, y) \geq 2 m+1$. To show that this is in fact an equality, we construct a path from $x$ to $y$ in $\mathcal{F}(G, X)$ as follows, using Lemma 2.3 to verify adjacency at each step:

$$
\begin{aligned}
& x=(\stackrel{+}{1}, \stackrel{+}{2})(\overline{3})(\overline{4}) \cdots\left(m_{+}^{-} 2\right)\left(m_{+}^{+} 3\right) \cdots\left(2 m^{+}+2\right), \\
& z_{1}=(\stackrel{+}{1}, m+3)(\stackrel{+}{2})(\overline{3})(\overline{4}) \cdots\left(m_{+}^{-} 2\right)\left(m_{+}^{+} 4\right) \cdots\left(2 m^{+}+2\right) \text {, } \\
& z_{2}=(\stackrel{+}{1}, \stackrel{+}{3})(\stackrel{+}{2})(\overline{4}) \cdots(m+3)\left(m_{+}^{+} 4\right) \cdots\left(2 m^{+}+2\right), \\
& z_{3}=\left(\stackrel{+}{1}, m_{+}^{+} 4\right)(\stackrel{+}{2})(\stackrel{+}{3})(\overline{4}) \cdots(m+3)\left(m^{+}+5\right) \cdots\left(2 m^{+}+2\right) \text {, } \\
& z_{4}=(\stackrel{+}{1}, \stackrel{+}{4})(\stackrel{+}{2})(\stackrel{+}{3})(\overline{5}) \cdots\left(m_{+}^{+} 4\right)\left(m_{+}^{+} 5\right) \cdots\left(2 m^{+}+2\right), \\
& \text { : } \\
& z_{2 m}=(\stackrel{+}{1}, m+2)(\stackrel{+}{2})(\stackrel{+}{3}) \cdots(m+\stackrel{+}{+})(m \stackrel{-}{+} 3) \cdots\left(2 m^{-}+2\right), \\
& y=(\stackrel{\epsilon}{1}, \stackrel{\epsilon}{2})(\stackrel{+}{3})(\stackrel{+}{4}) \cdots\left(m^{+}+2\right)(m+3) \cdots\left(2 m^{-}+2\right) .
\end{aligned}
$$

Since this path has length $2 m+1$, we deduce that $d(x, y)=2 m+1$.

Now assume that $\left|\Delta_{x, y}\right|=3$ and $w_{1}(x y)=k$. Here it need not be the case that $k$ is even. If $k=0$ the result follows by Lemma 2.3, so assume that $k \geq 1$. Thus $n \geq 4$. Using vertex-transitivity and Proposition 2.1 we see that, up to relabelling, there are the following possibilities for $x$ and $y$ :

(i) $k=2 m$,

$$
x=(\stackrel{+}{1}, \stackrel{+}{2})(\stackrel{\delta}{3})(\overline{4}) \cdots(m+3)\left(m^{+}+4\right) \cdots\left(2 m^{+}+3\right)
$$


and

$$
y=(\stackrel{\epsilon}{1}, \stackrel{\epsilon}{3}) \stackrel{\delta}{2})(\stackrel{+}{4}) \cdots(m \stackrel{+}{+} 3)(m-4+4) \cdots\left(2 m^{-}+3\right) ;
$$

(ii) $k=2 m+1$,

$$
x=(\stackrel{+}{1}, \stackrel{\delta}{2})(\stackrel{\delta}{3})(\overline{4}) \cdots(m+3)\left(m^{-\delta}+4\right)(m+5) \cdots\left(2 m^{+}+4\right)
$$

and

$$
y=(\stackrel{\epsilon}{1}, \stackrel{\epsilon}{\epsilon})(\stackrel{-\delta}{2})(\stackrel{+}{4}) \cdots\left(m^{+}+3\right)\left(m^{\delta}+4\right)\left(m_{+}^{-} 5\right) \cdots\left(2 m^{-}+4\right),
$$

where $\epsilon, \delta \in\{+,-\}$. In case (i), Lemma 2.4 implies that $d(x, y) \geq 2 m+1$, while in case (ii), Lemma 2.4 implies that $d(x, y) \geq 2 m+2$. However, we may construct paths from $x$ to $y$ in $\mathcal{F}(G, X)$ of length $2 m+1$ and $2 m+2$, in each case respectively. Since the method used to construct these paths is very similar in each case, we simply illustrate case (ii) where $\delta=+$. Here a suitable path is

$$
\begin{aligned}
& x=(\stackrel{+}{1}, \stackrel{+}{2})(\stackrel{+}{3})(\overline{4}) \cdots(m-4)\left(m_{+}^{+} 5\right) \cdots\left(2 m^{+}+4\right), \\
& z_{1}=\left(\stackrel{+}{1},+\frac{4}{4}\right)(\overline{2})(\stackrel{+}{3})(\overline{5}) \cdots(m+4)(m++5) \cdots\left(2 m^{+}+4\right), \\
& z_{2}=(\stackrel{+}{1}, m+5)(\overline{2})(\stackrel{+}{3})(\stackrel{+}{4})(\overline{5}) \cdots(m+4)\left(m^{+}+6\right) \cdots\left(2 m^{+}+4\right),
\end{aligned}
$$

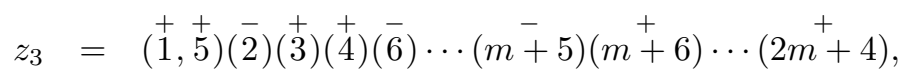

$$
\begin{aligned}
& z_{4}=\left(\stackrel{+}{1}, m_{+}^{+} 6\right)(\overline{2})(\stackrel{+}{3})(\stackrel{+}{4})(\stackrel{+}{5})(\overline{6}) \cdots\left(m_{+}^{-} 6\right)\left(m_{+}^{+} 7\right) \cdots\left(2 m^{+}+4\right), \\
& z_{2 m}=\left(\stackrel{+}{1}, 2 m^{+}+4\right)(\overline{2})(\stackrel{+}{3}) \cdots\left(m^{+}+3\right)\left(m_{+}^{-} 4\right) \cdots\left(2 m^{-}+4\right), \\
& z_{2 m+1}=(\stackrel{+}{1}, m+4)(\overline{2})(\stackrel{+}{3}) \cdots\left(m_{+}^{+} 3\right)\left(m_{-}^{-} 5\right) \cdots\left(2 m^{-}+4\right), \\
& y=(\stackrel{\epsilon}{1,3})(\overline{2})(\stackrel{+}{4}) \cdots\left(m^{+}+4\right)\left(m_{+}^{-} 5\right) \cdots\left(2 m^{-}+4\right) .
\end{aligned}
$$

Lemma 2.6. Suppose $x, y \in X$ with $\left|\Delta_{x, y}\right|=4$. Then there exists $z \in X$ such that $d(z, y)=1$ and either

(i) $\left|\Delta_{x, z}\right|=3$ and $w_{1}(x z)=w_{1}(x y)$; or

(ii) $\left|\Delta_{x, z}\right|=4$ and $w_{1}(x z)=w_{1}(x y)-1$.

Furthermore, $x$ is connected to $y$ in $\mathcal{F}(G, X)$, and any shortest path from $x$ to $y$ through $z$ has length $w_{1}(x y)+2$.

Proof. Write $w_{1}(x y)=k$. When $k=0$ it may be easily checked that an element $z \in X$ exists which satisfies (i), and that $d(x, y)=2$. Hence we may assume that $k \geq 1$, and so $n \geq 5$. As $y$ is certainly adjacent to some element $z^{\prime} \in X$ where $\left|\Delta_{x, z^{\prime}}\right|=3$, Lemma 2.5 implies that $y$ is connected to $x$ in $\mathcal{F}(G, X)$. 
Without loss of generality we may label $x$ and $y$ so that

$$
x=(\stackrel{+}{1}, \stackrel{+}{2})(\stackrel{\tau}{3}) \stackrel{\kappa}{4})(5)(6) \cdots(n)
$$

and

$$
y=(\stackrel{\mu}{1})(\stackrel{\nu}{2})(\stackrel{\epsilon}{3}, \stackrel{\epsilon}{4})(5)(6) \cdots(n),
$$

where $\tau, \kappa, \mu, \nu, \epsilon \in\{+,-\}$, and we make no assumption on the signs of the unlabelled cycles of $x$ and $y$. First suppose that $\{\tau, \kappa\} \cap\{\mu, \nu\} \neq \emptyset$, with say $\nu=\tau$. Let

$$
z=(\stackrel{\mu}{1})(\stackrel{\epsilon}{2} \stackrel{\epsilon}{4})(\stackrel{\tau}{)})(5)(6) \ldots(n) .
$$

Then $d(z, y)=1$ by Lemma 2.3, $w_{1}(x z)=w_{1}(x y)$ and $\left|\Delta_{x, z}\right|=3$, so $z$ satisfies (i).

Now suppose that $\{\tau, \kappa\} \cap\{\mu, \nu\}=\emptyset$. Then we must have $\tau=\kappa$ and $\mu=\nu=-\tau$, so we may without loss of generality assume that $x$ and $y$ are labelled so that

$$
x=(\stackrel{+}{1}, \stackrel{+}{2})(\stackrel{\tau}{3})(\stackrel{\tau}{4})(5)(6) \ldots(n)
$$

and

$$
y=(\stackrel{-\tau}{1})(\stackrel{-\tau}{2})(\stackrel{\epsilon}{\epsilon}, \stackrel{\epsilon}{4})(5)(6) \ldots(n) .
$$

By Proposition 2.1, there must exist $i, j \in\{5, \ldots, n\}$ such that $i \neq j, y$ contains the cycles $(\stackrel{\tau}{i})$ and $(\stackrel{\tau}{j})$, and $x$ contains the cycles $(\stackrel{-\tau}{i})$ and $(\stackrel{-\tau}{j})$ (and so $n \geq 6$ ). Without loss we may label $x$ and $y$ so that

$$
x=(\stackrel{+}{1}, \stackrel{+}{2})(\stackrel{\tau}{3})(\stackrel{\tau}{4})(\stackrel{-\tau}{5})\left(\stackrel{-}{6}^{-}\right) \ldots(n)
$$

and

$$
y=\left(\stackrel{-}{1}^{\tau}\right)(\stackrel{-\tau}{2})(\stackrel{\epsilon}{\epsilon}, \stackrel{\epsilon}{4})\left(\begin{array}{c}
\tau \\
5
\end{array}\right)(\stackrel{\tau}{6}) \ldots(n)
$$

Let

$$
z=\left(\begin{array}{c}
-\tau \\
1
\end{array}\right)(\stackrel{-\tau}{2})(\stackrel{\epsilon}{\epsilon}, \stackrel{\epsilon}{\epsilon})(\stackrel{\tau}{\tau})(\stackrel{\tau}{6}) \ldots(n) .
$$

Then $d(z, y)=1$ by Lemma $2.3,\left|\Delta_{x, z}\right|=4$ and $w_{1}(x z)=w_{1}(x y)-1$, so $z$ satisfies (ii).

To prove the final statement of the lemma we use induction on $k$. We have already noted that the lemma holds when $k=0$. If $z$ satisfies (i), then by Lemma 2.5 we have $d(x, z)=w_{1}(x z)+1=w_{1}(x y)+1$, and as $d(z, y)=1$ this implies that a shortest path from $x$ to $y$ through $z$ must have length $w_{1}(x y)+2$. On the other hand, if $z$ satisfies (ii) then $w_{1}(x z)=w_{1}(x y)-1$. Hence we may apply induction to see that $d(x, z)=w_{1}(x y)+1$, and again a shortest path from $x$ to $y$ through $z$ must have length $w_{1}(x y)+2$, as required to complete the proof.

Lemma 2.7. Suppose $x, y \in X$ with $\left|\Delta_{x, y}\right|=4$. Then $d(x, y)=w_{1}(x y)+2$. 
Proof. By Lemma 2.6, $y$ is connected to $x$ in $\mathcal{F}(G, X)$, so let $\gamma$ be a shortest path from $x$ to $y$, with $z \in \gamma$ such that $d(z, y)=1$. Using Lemma 2.3 there are potentially five possibilities for $z$ :

(i) $\left|\Delta_{x, z}\right|=3$ and $w_{1}(x z)=w_{1}(x y)$;

(ii) $\left|\Delta_{x, z}\right|=3$ and $w_{1}(x z)=w_{1}(x y)+1$;

(iii) $\left|\Delta_{x, z}\right|=4$ and $w_{1}(x z)=w_{1}(x y)-1$

(iv) $\left|\Delta_{x, z}\right|=4$ and $w_{1}(x z)=w_{1}(x y)$; or

(v) $\left|\Delta_{x, z}\right|=4$ and $w_{1}(x z)=w_{1}(x y)+1$.

In view of Lemma 2.6 it suffices to show that the possibilities (ii), (iv) and (v) cannot occur.

First, suppose that (ii) holds. Then by Lemma 2.4 we have $d(x, z) \geq$ $w_{1}(x y)+2$, which implies $d(x, y) \geq w_{1}(x y)+3$, contradicting Lemma 2.6. Next, suppose (iv) holds. Then $z$ must lie in the same $C_{G}(x)$-orbit as $y$, which contradicts $\gamma$ being a shortest path using Lemma 2.2. Finally, suppose (v) holds. Then by Lemma 2.4 we have $d(x, z) \geq w_{1}(x z)+2$, which implies $d(x, y) \geq w_{1}(x y)+3$, again contradicting Lemma 2.6. Thus either (i) or (iii) must hold, and the result follows by the final statement of Lemma 2.6 .

Taken together, Lemmas 2.5 and 2.7 shows that the local fusion graphs of $C\left(B_{n}\right)$ which arise from conjugacy classes of signed transpositions are connected. We can now prove the following result regarding the diameters of these local fusion graphs.

Theorem 2.8. Let $G=C\left(B_{n}\right)$, where $n \geq 4$.

(i) If $n$ is even, then there exist $G$-conjugacy classes of signed transpositions $X_{1}$ and $X_{2}$ such that $\operatorname{Diam} \mathcal{F}\left(G, X_{1}\right)=n-1$ and $\operatorname{Diam} \mathcal{F}\left(G, X_{2}\right)=n-2$. Moreover, if $Y$ is any other $G$-conjugacy class of signed transpositions, then $\operatorname{Diam} \mathcal{F}(G, Y) \leq n-1$.

(ii) If $n$ is odd, then there exists a $G$-conjugacy class of signed transpositions $X_{3}$ such that $\operatorname{Diam} \mathcal{F}\left(G, X_{3}\right)=n-2$. Moreover, if $Y^{\prime}$ is any other $G$ conjugacy class of signed transpositions, then $\operatorname{Diam} \mathcal{F}\left(G, Y^{\prime}\right) \leq n-2$.

Proof. Suppose that $n$ is even, and write $n=2 m$. Set $X_{1}=x_{1}^{G}$, where

$$
x_{1}=(\stackrel{+}{1}, \stackrel{+}{2})(\stackrel{+}{3})(\stackrel{+}{4})(\overline{5}) \cdots(m+2)(m+3) \cdots(\stackrel{+}{+}) .
$$

If we now let

$$
y_{1}=(\stackrel{+}{1})(\stackrel{+}{2})(\stackrel{+}{3}, \stackrel{+}{4})(\stackrel{+}{5}) \cdots\left(m_{+}^{+} 2\right)\left(m_{-}^{-} 3\right) \cdots\left(2 m^{-}\right),
$$

then $\left|\Delta_{x_{1}, y_{1}}\right|=4$ and $w_{1}(x y)=2 m-4$, so Lemma 2.7 tells us that

$$
d\left(x_{1}, y_{1}\right)=2 m-2=n-2 .
$$


Since it is impossible to choose $y_{1}^{\prime} \in X_{1}$ where $w_{1}\left(x_{1} y_{1}^{\prime}\right)>w_{1}\left(x_{1} y_{1}\right)$, Lemmas 2.5 and 2.7 show that this distance is maximal in $\mathcal{F}\left(G, X_{1}\right)$, whence $\operatorname{Diam} \mathcal{F}\left(G, X_{1}\right)=$ $n-2$.

Next, set $X_{2}=x_{2}^{G}$, where

$$
x_{2}=(\stackrel{+}{1}, \stackrel{+}{2})(\overline{3}) \cdots(m \stackrel{-}{+})(m \stackrel{+}{+} 2) \cdots(2 \stackrel{+}{m}) .
$$

If we let

$$
y_{2}=(\stackrel{+}{1}, \stackrel{+}{2})(\stackrel{+}{3}) \cdots(m \stackrel{+}{+} 1)(m+2) \cdots(2 \bar{m}),
$$

then $\left|\Delta_{x_{2}, y_{2}}\right|=2$ and $w_{1}\left(x_{2} y_{2}\right)=2 m-2$, so by Lemma 2.5 we have $d\left(x_{2}, y_{2}\right)=$ $2 m-1=n-1$. It is impossible to choose $y_{2}^{\prime} \in X_{2}$ such that $w_{1}\left(x_{2} y_{2}^{\prime}\right)>$ $w_{1}\left(x_{2} y_{2}\right)$, and if $y_{2}^{\prime}$ is such that $\left|\Delta_{x_{2}, y_{2}^{\prime}}\right|=4$ then we must have $w_{1}\left(x_{2} y_{2}^{\prime}\right)<$ $w_{1}\left(x_{2} y_{2}\right)$. Therefore, using Lemmas 2.5 and 2.7 we deduce that $y_{2}$ is an element of $X_{2}$ at maximal distance from $x_{2}$ in $\mathcal{F}\left(G, X_{2}\right)$, and so $\operatorname{Diam} \mathcal{F}\left(G, X_{2}\right)=n-1$. To see the final statement of (i), note that if $x^{\prime}$ and $y^{\prime}$ are signed transpositions in any other $G$-conjugacy class of signed transpositions $Y$, then $w_{1}\left(x^{\prime} y^{\prime}\right)<2 m-2$, so by Lemmas 2.5 and 2.7 we have $d\left(x^{\prime}, y^{\prime}\right) \leq 2 m-1=n-1$.

Now suppose that $n=2 m+1$ and set $X_{3}=x_{3}^{G}$, where

$$
x_{3}=(\stackrel{+}{1}, \stackrel{+}{2})(\stackrel{+}{3})(\overline{4})(\overline{5}) \cdots(m-2)\left(m_{+}^{+} 3\right) \cdots\left(2 m^{+}+1\right) .
$$

If we let

$$
y_{3}=(\stackrel{+}{1})(\stackrel{+}{2})(\stackrel{+}{3}, \stackrel{+}{4})(\stackrel{+}{5}) \cdots(m \stackrel{+}{+} 2)(m-\overrightarrow{+} 3) \cdots\left(2 m^{-}+1\right),
$$

then $\left|\Delta_{x_{3}, y_{3}}\right|=4$ and $w_{1}\left(x_{3} y_{3}\right)=2 m-3$, so by Lemma 2.7 we have $d\left(x_{3}, y_{3}\right)=$ $2 m-1=n-2$. It is possible to choose $y_{3}^{\prime} \in X_{3}$ such that $w_{1}\left(x_{3} y_{3}^{\prime}\right)=2 m-2$, however to do so we must have $\left|\Delta_{x_{3}, y_{3}^{\prime}}\right|=2$ or 3 . Thus, using Lemmas 2.5 and 2.7 we deduce that $y_{3}$ is at maximal distance from $x_{3}$ in $\mathcal{F}(G, X)$, which yields $\operatorname{Diam} \mathcal{F}\left(G, X_{3}\right)=n-2$. For the final statement of (ii), notice that for $x^{\prime}$ and $y^{\prime}$ in any other $G$-conjugacy class of signed transpositions $Y^{\prime}$, then either $w_{1}\left(x^{\prime} y^{\prime}\right) \leq n-4$, or $w_{1}\left(x^{\prime} y^{\prime}\right)=n-3$ and $\left|\Delta_{x^{\prime}, y^{\prime}}\right|=2$ or 3 . In both cases, by Lemmas 2.5 and 2.7 we have $\operatorname{Diam} \mathcal{F}\left(G, Y^{\prime}\right) \leq n-2$.

The case where $n=3$ is excluded from Theorem 2.8 since for $G=C\left(B_{3}\right)$ and $X=x^{G}$, where $x=(\stackrel{+}{1}, \stackrel{+}{2})(\stackrel{+}{3})$ or $(\stackrel{+}{1}, \stackrel{+}{2})(\overline{3})$, we have $\operatorname{Diam} \mathcal{F}(G, X)=2$. Note that Theorem 2.8 partially proves Theorem 1.4 , by showing the existence of local fusion graphs with diameter $r$ for all $r \geq 3$. Since the existence of local fusion graphs with diameters 1 and 2 is clear, to complete the proof of Theorem 1.4 we just need to show that any number of connected components is possible. This is resolved by our next result.

Lemma 2.9. Let $H$ be a finite group, with $X$ an $H$-conjugacy class of involutions, and suppose $\mathcal{F}(H, X)$ is connected. Let $L=H$ l $\operatorname{Sym}(m)$. Then there exists an L-conjugacy class of involutions $Y$ such that $\mathcal{F}(L, Y)$ has exactly $m$ connected components, each isomorphic to $\mathcal{F}(H, X)$. 
Proof. The wreath product $L=H \curlywedge \operatorname{Sym}(m)$ has base group $H_{1} \times \cdots \times H_{m}$, where $H_{i} \cong H$ for $1 \leq i \leq m$. Let $Y$ be the $L$-conjugacy class which contains the canonical image of $X$ in $H_{1}$. Since $\operatorname{Sym}(m)$ acts transitively on the components of the base group, $Y$ can be considered as a union of $m$ copies of $X$. Since elements of $Y$ from distinct components commute, they can never be adjacent in $\mathcal{F}(L, Y)$. It follows that $\mathcal{F}(L, Y)$ has $m$ connected components, each of which is isomorphic to $\mathcal{F}(H, X)$.

Combining Lemma 2.9 with Theorem 2.8 now yields Theorem 1.4. Having considered the case of signed transpositions, we now move to the opposite extreme, those conjugacy classes of involutions which have at most one 1-cycle. First we collect together some data for cases when the rank $n$ is small, which will be used in proving the more general results which follow.

Lemma 2.10. Suppose $G=C\left(B_{n}\right)$, where $5 \leq n \leq 10$.

(i) If $n=2 m$, set $X=t^{G}$ where

$$
t=(\stackrel{+}{1}, \stackrel{+}{2}) \ldots\left(2 m^{+}-1,2 \stackrel{+}{m}\right) .
$$

Then $\mathcal{F}(G, X)$ has exactly two connected components. When $n=8$ these components have diameter 3 , while if $n=6$ or 10 this diameter is 2 .

(ii) If $n=2 m+1$, set $X=t_{\epsilon}^{G}$ where

$$
t_{\epsilon}=(\stackrel{+}{1}, \stackrel{+}{2}) \ldots\left(2 m^{+}-1,2 \stackrel{+}{m}\right)(2 m \stackrel{\epsilon}{+} 1)
$$

and $\epsilon \in\{+,-\}$. Then $\mathcal{F}(G, X)$ is connected, and $\operatorname{Diam} \mathcal{F}(G, X)=2$.

Proof. Since these local fusion graphs have relatively small vertex sets, it is straightforward to explicitly construct them using MAGMA [7], for example.

Lemma 2.11. Let $G=C\left(B_{n}\right)$, and suppose $\sigma \in G$ is a signed cycle. Then $\sigma$ has odd order if, and only if,

(i) the length of $\sigma$ is odd; and

(ii) the weight of $\sigma$ is even.

Proof. Without loss of generality suppose that, without signs, $\sigma=(1,2, \ldots, k)$. It is clearly the case that $\sigma$ either has order $k$ or $2 k$. Thus for $\sigma$ to have odd order it is necessary that (i) holds, so suppose that $k$ is odd. If $w(\sigma)=0$ then certainly $\sigma$ has odd order $k$. Suppose that $w(\sigma)=0$, and let $\sigma_{1}$ be equal to $\sigma$ multiplied by a single negative 1-cycle, say

$$
\sigma_{1}=(\bar{i}) \sigma
$$

where $i \in\{1, \ldots, k\}$. Then $w\left(\sigma_{1}\right)=1$. Moreover, we have

$$
\sigma_{1}^{k}=(\overline{1})(\overline{2}) \ldots(\bar{k})
$$


and so $\sigma_{1}$ has order $2 k$. If we now multiply $\sigma_{1}$ by a negative 1 -cycle, say

$$
\sigma_{2}=(\bar{j}) \sigma_{1}
$$

where $j \in\{1, \ldots, k\}$, then we have $w\left(\sigma_{2}\right)=0$ or 2 , and we get

$$
\sigma_{2}^{k}=(\overline{1})(\overline{2}) \ldots(\bar{k}) \sigma_{1}^{k}=1
$$

so $\sigma_{2}$ has order $k$. More generally, if we multiply $\sigma$ by an odd number of negative 1 -cycles, the resulting product will have odd weight and order $2 k$, while if we multiply $\sigma$ by an even number of negative 1-cycles, the resulting product will have even weight and order $k$. Since it is possible to reach any signed $k$-cycle in $G$ by multiplying a $k$-cycle of weight zero by a number of negative 1 -cycles, we deduce that if $w(\sigma)$ is even the order of $\sigma$ is $k$, while if $w(\sigma)$ is odd the order of $\sigma$ is $2 k$.

Lemma 2.12. Suppose $H=\operatorname{Sym}(2 m)$ acts naturally on $\Omega$, and set $X=x^{H}$, where

$$
x=(1,2)(3,4) \cdots(2 m-1,2 m) .
$$

If $y \in X$, then $y$ is adjacent to $x$ in $\mathcal{F}(H, X)$ if, and only if,

$$
x y=\sigma_{1,1} \sigma_{1,2} \sigma_{2,1} \sigma_{2,2} \ldots \sigma_{s, 1} \sigma_{s, 2}
$$

is a product of pairwise disjoint cycles, where for $1 \leq r \leq s$ the cycles $\sigma_{r, 1}$ and $\sigma_{r, 2}$ have the same odd length. Moreover, if $i, j \in \Omega$ lie in the same transposition of $y$, then we can label the cycles of $x y$ so that $i \in \sigma_{1,1}$ and $j \in \sigma_{1,2}$.

Proof. This follows from Proposition 2.2 of [6].

We now consider $C\left(B_{n}\right)$ for arbitrary rank $n \geq 3$, and address the connectedness and diameter of those local fusion graphs which have as vertices involutions which contain at most one 1-cycle. In preparation we introduce another piece of notation. Given $g \in C\left(B_{n}\right)$, we denote by $\bar{g}$ the element of $\operatorname{Sym}(n)$ we get by ignoring all signs of $g$. For example, if $g \in C\left(B_{6}\right)$ and

$$
g=(\stackrel{+}{1}, \overline{2}, \stackrel{+}{3})(\overline{4}, \overline{5})(\stackrel{+}{6})
$$

then

$$
\bar{g}=(1,2,3)(4,5)(6) .
$$

Lemma 2.13. Suppose $G=C\left(B_{2 m}\right)$, where $m \geq 3$, and let

$$
t=(\stackrel{+}{1}, \stackrel{+}{2})(\stackrel{+}{3}, \stackrel{+}{4}) \ldots\left(2 m^{+}-1,2 \stackrel{+}{m}\right),
$$

with $X=t^{G}$. Then $\mathcal{F}(G, X)$ has exactly two connected components, one containing all $x \in X$ with $w(x) \equiv 0 \bmod 4$, the other containing all $x \in X$ with $w(x) \equiv 2 \bmod 4$. Furthemore, the diameter of each component is at most 5. 
Proof. Note that Proposition 2.1 implies that all elements of $X$ have even weight. Let $\bar{G}=\operatorname{Sym}(2 m)$, and denote by $\bar{X}$ the $\bar{G}$-conjugacy class which contains the element $\bar{t}$. Suppose that $x \in X$ is adjacent to $t$ in $\mathcal{F}(G, X)$. Then $t x$ must be a product of pairwise disjoint, odd-length signed cycles, each of which has even weight by Lemma 2.11. If $(\bar{i}, \bar{j})$ is a transposition in $x$, then, since $\bar{t}$ and $\bar{x}$ must be adjacent in $\mathcal{F}(\bar{G}, \bar{X})$, we may use Lemma 2.12 to see that $\bar{i}$ and $\bar{j}$ lie in distinct cycles of $t x$. Therefore for every cycle of $t x$ to have even weight it must be that $x$ has an even number of transpositions with weight 2 , and so $w(x) \equiv w(t) \bmod 4$. Since $G$ acts vertex-transitively on $\mathcal{F}(G, X)$ we have that every element of the connected component of $\mathcal{F}(G, X)$ which contains $t$ has the same weight as $t$ modulo 4 .

Now suppose that $x \in X$ with $w(x) \equiv w(t) \bmod 4$. Since Lemma 2.10 completes the proof for $m<6$, we may assume that $m \geq 6$. By Theorem 1.1 of [1], $\mathcal{F}(\bar{G}, \bar{X})$ has diameter 2 , so there exists some element $z \in X$ such that $\bar{x}=\bar{z}$, and $d(t, z) \leq 2$. We may arrange the (pairwise disjoint) 2-cycles of $x$ and $z$ so that

$$
x=x^{(1)} x^{(2)} \ldots x^{(r)}
$$

and

$$
z=z^{(1)} z^{(2)} \ldots z^{(r)},
$$

and the following conditions are satisfied:

(i) the $x^{(i)}$ are products of three signed 2-cycles, except for possibly $x^{(r)}$ which may be a product of four or five signed 2-cycles;

(ii) $\overline{x^{(i)}}=\overline{z^{(i)}}$ for each $i$; and

(iii) $w\left(x^{(i)}\right) \equiv w\left(z^{(i)}\right) \bmod 4$, for each $i$.

Considering each pair $x^{(i)}, z^{(i)}$ as elements of $C\left(B_{6}\right)$ (or possibly $C\left(B_{8}\right)$ or $C\left(B_{10}\right)$ for the final pair), we now apply Lemma 2.10 to see that for each $i$ there exist paths from $z^{(i)}$ to $x^{(i)}$ of length at most 3 , in the relevant local fusion graphs of $C\left(B_{6}\right), C\left(B_{8}\right)$ or $C\left(B_{10}\right)$. Since both $x^{(i)}$ and $z^{(i)}$ are disjoint from all other $x^{(j)}, z^{(j)}$ (where $i \neq j$ ), by taking products of suitable elements from each such path, we may construct a path of length at most 3 from $z$ to $x$ in $\mathcal{F}(G, X)$. Thus we have a path from $t$ to $x$ of length at most 5 . It follows that the elements of $X$ with weight congruent to 0 modulo 4 form a connected component of $\mathcal{F}(G, X)$. Since this accounts for exactly half the elements of $X$, by the vertex-transitivity of $\mathcal{F}(G, X)$ we deduce that there are exactly two connected components, the second of which must consist of the elements of $X$ with weight congruent to 2 modulo 4 .

Lemma 2.14. Suppose $G=C\left(B_{2 m+1}\right)$, where $m \geq 2$, and let

$$
t=(\stackrel{+}{1}, \stackrel{+}{2})(\stackrel{+}{3}, \stackrel{+}{4}) \ldots\left(2 m^{+}-1,2^{+} m\right)\left(2 m^{\epsilon}+1\right),
$$


where $\epsilon \in\{+,-\}$, with $X=t_{\epsilon}^{G}$. Then $\mathcal{F}(G, X)$ is connected, and

$$
\operatorname{Diam} \mathcal{F}(G, X) \leq 4
$$

Proof. Our argument here is similar to that of the previous proof, and we adopt the same notation. Let $x \in X$. By Theorem 1.1 of $[1], \mathcal{F}(\bar{G}, \bar{X})$ has diameter 2 , so there exists some element $z \in X$ such that $\bar{x}=\bar{z}$, and $d(t, z) \leq 2$. We may arrange the (pairwise disjoint) cycles of $x$ and $z$ so that

$$
x=x^{(1)} x^{(2)} \cdots x^{(r)}
$$

and

$$
z=z^{(1)} z^{(2)} \cdots z^{(r)}
$$

and the following conditions are satisfied:

(i) the $x^{(i)}$ are products of three signed 2-cycles, except for $x^{(r)}$ which is a product of two, three or four signed 2-cycles with a signed 1-cycle;

(ii) $\overline{x^{(i)}}=\overline{z^{(i)}}$ for each $i$; and

(iii) $w\left(x^{(i)}\right) \equiv w\left(z^{(i)}\right) \bmod 4$, for each $i<r$.

For $1 \leq i<r$, we may now consider each pair $x^{(i)}, z^{(i)}$ as elements of $C\left(B_{6}\right)$, while the pair $x^{(r)}, z^{(r)}$ may be considered as elements of $C\left(B_{5}\right), C\left(B_{7}\right)$ or $C\left(B_{9}\right)$. Using Lemma 2.10 we now have $d(z, x) \leq 2$, and consequently there exists a path from $t$ to $x$ in $\mathcal{F}(G, X)$ of length at most 4 .

Given an involution $x \in C\left(B_{\Omega}\right)$, and a subset $\Sigma \subset \Omega$, we denote by $x_{\Sigma}$ the element of $C\left(B_{\Sigma}\right)$ we get by omitting all signed cycles of $x$ which involve elements of $\Omega \backslash \Sigma$. For example, if $\Omega=\{1,2,3,4,5,6\}, \Sigma=\{1,2,5\}$ and

$$
x=(\overline{1}, \overline{2})(\stackrel{+}{3}, \stackrel{+}{4})(\overline{5})(\stackrel{+}{6}),
$$

then

$$
x_{\Sigma}=(\overline{1}, \overline{2})(\overline{5}) .
$$

We can now say something about the local fusion graphs of the remaining involution classes of $C\left(B_{n}\right)$.

Theorem 2.15. Let $G=C\left(B_{n}\right)$, where $n \geq 4$, and let $X$ be a $G$-conjugacy class of involutions where the elements of $X$ contain at least one 1-cycle. Then $\mathcal{F}(G, X)$ is connected, with

$$
\operatorname{Diam} \mathcal{F}(G, X) \leq n-1
$$

if $n$ is even, and

$$
\operatorname{Diam} \mathcal{F}(G, X) \leq n-2
$$

if $n$ is odd. 
Proof. When $n<10$ this can be verified computationally using MAgma, so assume $n \geq 10$. Suppose $t, x \in X$, and without loss of generality assume that

$$
x=(\stackrel{+}{1}, \stackrel{+}{2}) \cdots\left(2 r_{-}^{+} 1,2 r\right)(2 r-\overrightarrow{+} 1) \cdots(\bar{s})\left(s_{+}^{+} 1\right) \cdots(\stackrel{+}{n}) .
$$

Since we have dealt with signed transpositions in Theorem 2.8, we may assume that $r \geq 2$. By Theorem 1.1 of [1] there exists some $y \in X$ such that $d(t, y) \leq 2$ and $\bar{y}=\bar{x}$, which without loss of generality we may label so that

$$
y=(1,2) \cdots(2 r-1,2 r)(2 r+1) \cdots\left(\begin{array}{c}
\epsilon_{2 r+1} \\
s
\end{array}\right)\left(\begin{array}{c}
\epsilon_{s+1} \\
s+1
\end{array}\right) \cdots\left(\begin{array}{c}
\epsilon_{n} \\
n
\end{array}\right),
$$

where $\epsilon_{i} \in\{+,-\}$ for $i \in\{2 r+1, \ldots, n\}$, and we make no assumption on the signs of the 2-cycles of $y$. Note that $w_{1}(x y)$ must be even by Proposition 2.1. Suppose that $x$ and $y$ differ by $2 k$ signed 1 -cycles. Since $r \geq 2$ it must be that $2 k \leq n-4$.

If $k=0$ then, by ignoring all but one 1-cycle of $x$ and $y$, we may apply Lemma 2.14 to see that $d(y, x) \leq 4$, and so $d(t, x) \leq 2+4=6$, which suffices since we have assumed $n \geq 10$.

Next, suppose that $k=1$. If we ignore all 2-cycles of $x$ and $y$ except $(1,2)$, we can consider the resulting elements $\tilde{x}$ and $\tilde{y}$ as signed transpositions in $K=C\left(B_{\Sigma}\right)$, where $\Sigma=\Omega \backslash\{3, \ldots, 2 r\}$. By Lemma 2.5 there exists a path in $\mathcal{F}(K, \tilde{X})$ from $\tilde{y}$ to $\tilde{x}$ (where $\tilde{X}$ is the $K$-conjugacy class which contains $\tilde{x}$ ), which has length 3 . This induces a path of length 3 in $\mathcal{F}(G, X)$ from $y$ to an element $z \in X$ such that $\bar{z}=\bar{x}$, and $z$ and $x$ agree on all signed 1-cycles. Now, ignoring all but one 1-cycle of $z$ and $x$, we may apply Lemma 2.14 to deduce that $d(z, x) \leq 4$. Thus $d(t, x) \leq 2+3+4=9$, and again the result holds.

Now assume that $k \geq 2$. Since $r \geq 2$ we may also assume that both $x$ and $y$ contain the 2 -cycles $(1,2)$ and $(3,4)$. We may partition $\operatorname{supp}_{C}(x y) \backslash \Delta_{x, y}$ into two subsets $A$ and $B$, such that the following conditions hold:

(i) either $|A|=|B|=k$, or $|A|=k+1$ and $|B|=k-1$; and

(ii) if we write $K_{1}=C\left(B_{\Sigma_{1}}\right)$ and $K_{2}=C\left(B_{\Sigma_{2}}\right)$, where $\Sigma_{1}=\{1,2\} \cup A$ and $\Sigma_{2}=\{3,4\} \cup B$, then $x_{\Sigma_{i}}$ and $y_{\Sigma_{i}}$ are $K_{i}$-conjugate, for $i=1,2$.

In view of Proposition 2.1, condition (ii) above is equivalent to requiring that the number of negative 1-cyles in $x_{\Sigma_{i}}$ be equal to the number of negative 1cycles in $y_{\Sigma_{i}}$. Since both $\Sigma_{1}$ and $\Sigma_{2}$ have cardinality at least 3 , we may apply Lemma 2.5 to see that there exist paths in $\mathcal{F}\left(K_{i}, X_{i}\right)$ from $y_{\Sigma_{i}}$ to $x_{\Sigma_{i}}$ (where $X_{i}$ is the $K_{i}$-conjugacy class which contains $x_{\Sigma_{i}}$, for $\left.i=1,2\right)$. Since for each $i$ we have $w_{1}\left(x_{\Sigma_{i}} y_{\Sigma_{i}}\right) \leq k+1$, these paths will be of length at most $k+2$. Furthermore, as all elements of $X_{1}$ fix $\Sigma_{2}$ pointwise, and all elements of $X_{2}$ fix $\Sigma_{1}$ pointwise, we may multiply elements from these paths together (along with any remaining signed cycles of $y$ ) to yield a path in $\mathcal{F}(G, X)$ of length at most $k+2$, between $y$ and an element $z \in X$ such that $\bar{z}=\bar{x}$, and $z$ and $x$ agree on all signed 1-cycles. By Lemma 2.14, $d(z, x) \leq 4$, so

$$
d(t, x) \leq 2+(k+2)+4=k+8 .
$$


First assume that $r \geq 4$. Then $2 k \leq n-8$, and hence $k \leq n / 2-4$. This yields

$$
d(t, x) \leq n / 2+4
$$

Now assume that $r=2$ or 3 . Here we may apply Lemma 2.10 to show that $d(z, x) \leq 2$, and so in this case we have

$$
d(t, x) \leq 2+(k+2)+2=k+6
$$

But $r \geq 2$, and so $2 k \leq n-4$ which implies $k \leq n / 2-2$. Consequently,

$$
d(t, x) \leq n / 2+4
$$

in this case also. Since $n \geq 10$, and $d(t, x)$ must be an integer, we have that $d(t, x) \leq n-1$ when $n$ is even, and $d(t, x) \leq n-2$ when $n$ is odd, as required.

Notice that the establishment of Theorem 2.15 completes the proofs of Theorems 1.1 and 1.3. We also have the following corollary, which completes the proof of Theorem 1.2.

Corollary 2.16. Let $G=C\left(D_{n}\right)$, with $X$ a $G$-conjugacy class of involutions whose elements contain at least one 2 -cycle. Then $\mathcal{F}(G, X)$ is connected, with $\operatorname{Diam}(\mathcal{F}(G, X) \leq n-1$ if $n$ is even, and $\operatorname{Diam} \mathcal{F}(G, X) \leq n-2$ if $n$ is odd.

Proof. This follows from Theorems 2.8 and 2.15, along with Lemma 2.13 and Proposition 2.1, which tells us that the conjugacy class of $C\left(B_{n}\right)$ for which the local fusion graph has two connected components splits into two classes in $G$, with local fusion graphs isomorphic to a connected component of the $C\left(B_{n}\right)$ graph.

\section{The Exceptional Groups}

We conclude by examining the local fusion graphs of the finite, exceptional Coxeter groups, and proving Theorem 1.5. The cases when $G=C\left(I_{n}\right)$ (the dihedral groups) are covered by the following lemma.

Lemma 3.1. Let $G=C\left(I_{n}\right)$. If $n$ is odd then there is exactly one conjugacy class of involutions, and the local fusion graph is the complete graph on $n$ vertices. If $n$ is even then $G$ has a central involution, and precisely two further conjugacy classes of involutions. If we write $n=2^{k} m$, where $m$ is odd, then each of these local fusion graphs has $n / 2$ vertices, with $2^{k-1}$ connected components, each component being a copy of the complete graph on $m$ vertices.

Proof. If $n$ is odd then it is straightforward to see that any product of two involutions must have odd order, so assume that $n=2^{k} m$ where $k \geq 1$ and $m$ is odd. We may present $G$ as

$$
G=\left\langle a, b \mid a^{2^{k} m}=b^{2}=1, b a b=a^{-1}\right\rangle .
$$


The non-trivial $G$-conjugacy classes of involutions are now

$$
\mathcal{I}_{e}=\left\{a^{i} b \mid 0 \leq i<2^{k} m, i \text { even }\right\}
$$

and

$$
\mathcal{I}_{o}=\left\{a^{i} b \mid 0 \leq i<2^{k} m, i \text { odd }\right\} .
$$

Suppose that $a^{i} b \in \mathcal{I}_{e}$. Then $a^{i} b$ is adjacent to $b$ in $\mathcal{F}\left(G, \mathcal{I}_{e}\right)$ if, and only if, $\left(a^{i} b\right) b=a^{i}$ has odd order, which occurs if, and only if, $2^{k}$ divides $i$. Hence there are $m$ choices for $i$, and the connected component of $\mathcal{F}\left(G, \mathcal{I}_{e}\right)$ which contains $b$ has $m$ vertices. Now we may use the vertex-transitivity of $G$ on $\mathcal{F}\left(G, \mathcal{I}_{e}\right)$ to deduce that there are $2^{k-1}$ connected components of $\mathcal{F}\left(G, \mathcal{I}_{e}\right)$, each of which is a copy of the complete graph on $m$ vertices. The proof for $\mathcal{F}\left(G, \mathcal{I}_{o}\right)$ is similar.

When $G=C\left(E_{6}\right), C\left(E_{7}\right), C\left(E_{8}\right), C\left(F_{4}\right), C\left(H_{3}\right)$ and $C\left(H_{4}\right)$, we proceed computationally. Representations of these groups are stored in MAGMA [7], and all are small enough to make explicit calculation of their local fusion graphs straightforward. Table 1 list the disc sizes for each local fusion graph of each group, along with representative involutions from each conjugacy class, given as words in the generators stored by MAGMA. 
Table 1: Disc sizes for exceptional Coxeter groups

\begin{tabular}{|c|c|c|c|c|c|}
\hline Group & Representative & Class size & $\left|\Delta_{1}(t)\right|$ & $\left|\Delta_{2}(t)\right|$ & $\left|\Delta_{3}(t)\right|$ \\
\hline$C\left(E_{6}\right)$ & $w_{1}$ & 36 & 20 & 15 & - \\
\hline$C\left(E_{6}\right)$ & $w_{0}$ & 45 & 32 & 12 & - \\
\hline$C\left(E_{6}\right)$ & $w_{1} w_{2}$ & 270 & 128 & 141 & - \\
\hline$C\left(E_{6}\right)$ & $w_{1} w_{2} w_{6}$ & 540 & 212 & 327 & - \\
\hline$C\left(E_{7}\right)$ & $w_{0}$ & 1 & - & - & - \\
\hline$C\left(E_{7}\right)$ & $w_{1}$ & 63 & 32 & 30 & - \\
\hline$C\left(E_{7}\right)$ & $w_{0} w_{1}$ & 63 & 32 & 30 & - \\
\hline$C\left(E_{7}\right)$ & $w_{2} w_{5} w_{7}$ & 315 & 128 & 186 & - \\
\hline$C\left(E_{7}\right)$ & $w_{0} w_{2} w_{5} w_{7}$ & 315 & 128 & 186 & - \\
\hline$C\left(E_{7}\right)$ & $w_{1} w_{2}$ & 945 & 416 & 528 & - \\
\hline$C\left(E_{7}\right)$ & $w_{0} w_{1} w_{2}$ & 945 & 416 & 528 & - \\
\hline$C\left(E_{7}\right)$ & $w_{0} w_{1} w_{2} w_{5} w_{7}$ & 3780 & 1568 & 2211 & - \\
\hline$C\left(E_{7}\right)$ & $w_{1} w_{2} w_{5} w_{7}$ & 3780 & 1568 & 2211 & - \\
\hline$C\left(E_{8}\right)$ & $w_{0}$ & 1 & - & - & - \\
\hline$C\left(E_{8}\right)$ & $w_{1}$ & 120 & 56 & 63 & - \\
\hline$C\left(E_{8}\right)$ & $w_{0} w_{1}$ & 120 & 56 & 63 & - \\
\hline$C\left(E_{8}\right)$ & $\left(w_{2} w_{3} w_{4} w_{5}\right)^{3}$ & 3150 & 512 & 2588 & 49 \\
\hline$C\left(E_{8}\right)$ & $w_{1} w_{2}$ & 3780 & 1472 & 2307 & - \\
\hline$C\left(E_{8}\right)$ & $w_{0} w_{1} w_{2}$ & 3780 & 1472 & 2307 & - \\
\hline$C\left(E_{8}\right)$ & $w_{1} w_{2} w_{5}$ & 37800 & 12344 & 25455 & - \\
\hline$C\left(E_{8}\right)$ & $w_{0} w_{1} w_{2} w_{5}$ & 37800 & 12344 & 25455 & - \\
\hline$C\left(E_{8}\right)$ & $w_{1} w_{2} w_{5} w_{7}$ & 113400 & 25280 & 88118 & 1 \\
\hline$C\left(F_{4}\right)$ & $w_{0}$ & 1 & - & - & - \\
\hline$C\left(F_{4}\right)$ & $w_{1}$ & 12 & 8 & 3 & - \\
\hline$C\left(F_{4}\right)$ & $w_{0} w_{1}$ & 12 & 8 & 3 & - \\
\hline$C\left(F_{4}\right)$ & $w_{3}$ & 12 & 8 & 3 & - \\
\hline$C\left(F_{4}\right)$ & $w_{0} w_{3}$ & 12 & 8 & 3 & - \\
\hline$C\left(F_{4}\right)$ & $\left(w_{2} w_{3}\right)^{2}$ & 18 & - & - & - \\
\hline$C\left(F_{4}\right)$ & $w_{1} w_{3}$ & 72 & 24 & 46 & 1 \\
\hline$C\left(H_{3}\right)$ & $w_{0}$ & 1 & - & - & - \\
\hline$C\left(H_{3}\right)$ & $w_{1}$ & 15 & 12 & 2 & - \\
\hline$C\left(H_{3}\right)$ & $w_{0} w_{1}$ & 15 & 12 & 2 & - \\
\hline$C\left(H_{4}\right)$ & $w_{0}$ & 1 & - & - & - \\
\hline$C\left(H_{4}\right)$ & $w_{1}$ & 60 & 44 & 15 & - \\
\hline$C\left(H_{4}\right)$ & $w_{0} w_{1}$ & 60 & 44 & 15 & - \\
\hline$C\left(H_{4}\right)$ & $w_{1} w_{3}$ & 450 & 168 & 280 & 1 \\
\hline
\end{tabular}




\section{References}

[1] J. Ballantyne, N. Greer, P. Rowley: Local Fusion Graphs for Symmetric Groups, J. Group Theory, 16 (2013) 35-49.

[2] J. Ballantyne, P. Rowley: Connectivity of Local Fusion Graphs for Finite Simple Groups, submitted.

[3] J. Ballantyne, P. Rowley: A Note on Computing Involution Centralizers, J. Symb. Comp. (2013), http://dx.doi.org/10.1016/j.jsc.2013.01.002.

[4] C. Bates, D. Bundy, S. Perkins, P. Rowley: Commuting involution graphs for finite Coxeter groups, J. Group Theory, 6 (2003) 461-476.

[5] C. Bates, D. Bundy, S. Perkins, P. Rowley: Commuting involution graphs for sporadic simple groups', J. Algebra, 316 (2007) 849-868.

[6] C. Bates, D. Bundy, S. Perkins, P. Rowley: Commuting involution graphs for symmetric groups, J. Algebra, 266 (2003) 133-153.

[7] W. Bosma, J. Cannon, C. Playoust: The Magma algebra system. I. The user language', J. Symbolic Comput., 24(3-4) (1997), 235-265.

[8] R.W. Carter: Conjugacy classes in the Weyl group, Compositio Math., 25 (1972) 1-59.

[9] J.H. Conway, R.T. Curtis, S.P. Norton, R.A. Parker, R.A. Wilson: Atlas of Finite Groups, Clarendon, Oxford (1985).

[10] A. Devillers, M. Giudici: Involution graphs where the product of two adjacent vertices has order three, J. Aust. Math. Soc., 85 (2008) 305-322.

[11] A. Devillers, M. Giudici, C. H. Li, C. E. Praeger: Some graphs related to the small Mathieu groups. European J. Combin. 31 (2010), no. 1, 336-348.

[12] B. Fischer: Finite groups generated by 3-transpositions, I. Invent. Math., 13 (1971), 232-246.

[13] G. Glauberman: Central elements in core-free groups, J. Algebra, 4 (1966) 403-420.

[14] D. Gorenstein: Finite groups, Second edition. Chelsea Publishing Co., New York (1980).

[15] J.E. Humphreys: Reflection groups and Coxeter groups, Cambridge Studies in Advanced Mathematics, 29. Cambridge University Press, Cambridge (1990). 